\title{
Flat-layered spherical lens antenna system in conditions of slant polarized feeder radiation
}

\author{
Ryazantsev R. O., Salomatov Y. P., Panko V.S. \\ Institute of Engineering Physics and Radio Electronics \\ Siberian Federal University \\ Krasnoyarsk, Russian Federation \\ rryazantsev@sfu-kras.ru
}

\begin{abstract}
Investigation results of flat-layered spherical lens made from homogenous dielectric material under illumination of slant polarized wave are presented. Lens anisotropy behavior impact on polarization characteristics of beam pattern main lobe is shown. Results of axial ratio simulation at beam pattern maximum direction in working frequency range are given for different polarization angles of incident wave and for different scan angles.
\end{abstract}

Keywords-Luneberg lens; lens antenna; circular polarization; beem steering.

\section{INTRODUCTION}

Luneberg lens [1] is well known as an antenna with the ability to perform wide angle scanning without changing of radiation pattern. The flat-layered spherical lens made from homogenous dielectric material [2-5] behaves like Luneburg Lens in wide angular range within certain frequency band if the incident wave polarization is linear.

However, if the lens is illuminated by a wave with a circular [6] or slant polarization the lens anisotropic properties appear more intensively. The aim of this paper is estimating of opportunities and limitations in using of flat-layered spherical lens as an antenna with elliptical polarization operating with a feed horn of slant linear polarization.

\section{LENS StRUCtURE AND PROBLEM FORMULATION}

The lens cross section is shown in Fig 1. This lens is a flatlayered spherical structure containing many disks of different size made of dielectric material with a relative dielectric constant equal to 1.6. In turn, discs are combined in layers of variable thickness. This approach allows creating a distribution of effective dielectric constant inside sphere as it occurs in the homogenous Luneberg lens [1]. The focal point of the lens is located at a distance $1.4 R$ from the sphere center, where $R$ is the sphere radius.

The described structure was studied at Q-band frequencies from 33 up to $50 \mathrm{GHz}$. Lens diameter was $90 \mathrm{~mm}$, the distance between centers of adjacent layers of variable thickness was $2.2 \mathrm{~mm}$. Thus, the lens diameter was approximately equal to $10 \lambda$ for the lower frequency.

\author{
Sugak M.I. \\ Faculty of Radio Engineering and Telecommunications \\ Saint-Petersburg State Electrotecnical University \\ Saint-Petersburg, Russia \\ sugakmi@yandex.ru
}

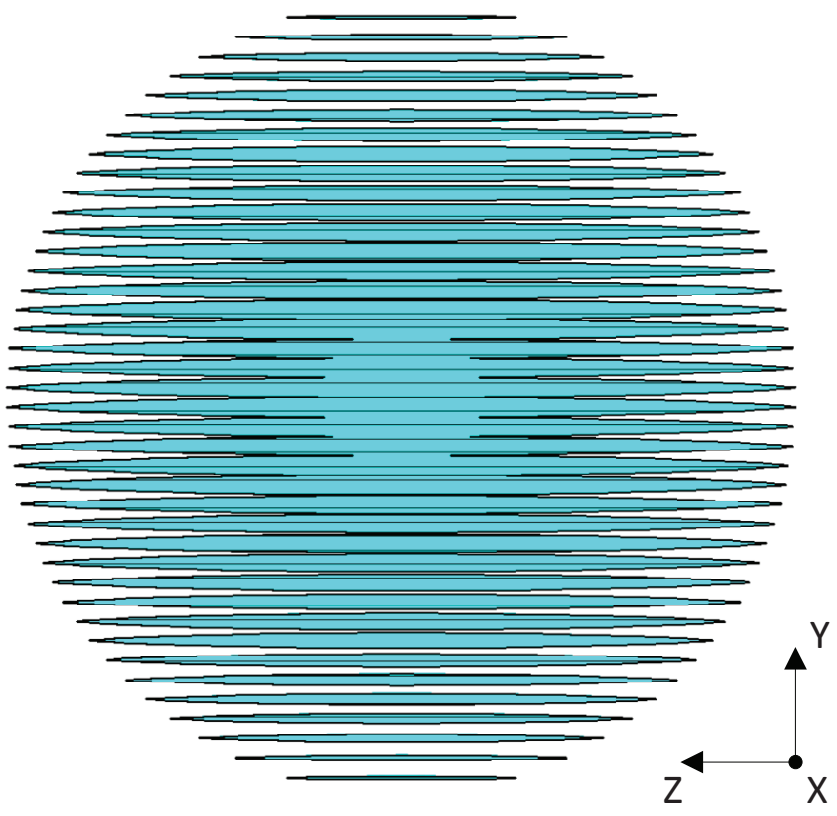

Fig. 1. Flat-layered spherical lens cross section.

When the electric vector of linearly polarized wave is perpendicular to the plane of layers of variable thickness and when the electric field vector lies in the plane of the layers, there is a difference in effective dielectric constant of lens structure. The difference is strongly dependent on the filling factor of the layer and depends only weakly on the maximum thickness of the layer of variable thickness, in other words, on the structure period [6]. The anisotropy of flat-layered structure imposes some restrictions on the permissible angles of scanning in $Y O Z$ plane, and obviously has an effect in the propagation of wave with circular [6] and slant polarizations through the lens.

The simulation results show that after illumination of a spherical flat-layered lens by a wave with linear polarization that is different from "vertical" (along the $Y$-axis) or "horizontal" (along the $X$-axis) the past wave polarization becomes elliptic. The reason for the observed effect is the effective dielectric permittivity difference for two kinds of wave polarizations. If the electric vector lies in plane with lens layers then the wave of such polarization will pass through the 
lens at different time in comparison with wave of "vertical" polarization.

The subject of our study is the influence of lens anisotropic properties on polarization characteristics of the radiation pattern main lobe for slant wave polarization.

For simulation a segment of circular waveguide with a round flange was employed as the feed horn. The flange comprises concentric cylindrical rings for obtaining of symmetric main beam. The feed horn model is shown in Fig. 2.

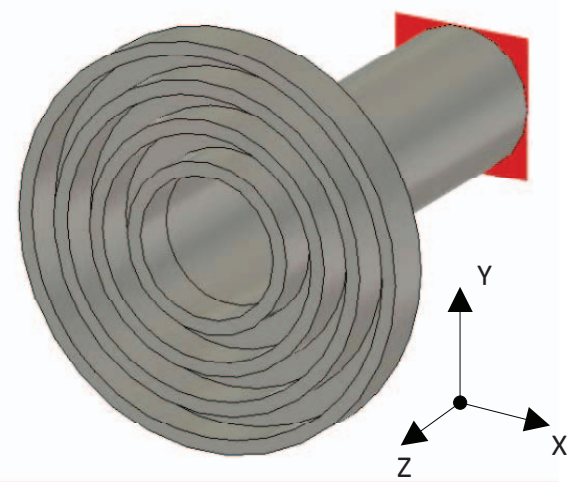

Fig. 2. Feed horn model isometric view for studying of the lens characteristics under slant wave polarization

Excitation of circular waveguide of the feed horn in this model was carried out by $H_{11}$ mode with different angles of polarization vector incline at the plane $X O Y$. The reference case is the polarization vector angle of $0^{\circ}$. In this case electric field vector lies along the $X$-axis in accordance with Fig. 1 and 2. The illuminating wave propagates along the $Z$ axis towards the lens and location of the feed horn phase center corresponds to the focal distance.

\section{Axial Ratio AND Polarization Plane ANGLES}

The results of calculations are obtained in full-wave EM software by finite integrals calculation in the time domain. The results show that the angle of the polarization plane affects the field axial ratio in the lens aperture. The dependence of axial ratio in the frequency range of interest is shown in Fig. 3 for different electric field vector incline angles. Angles are counted relative to "horizontal" orientation (the lens layers plane).

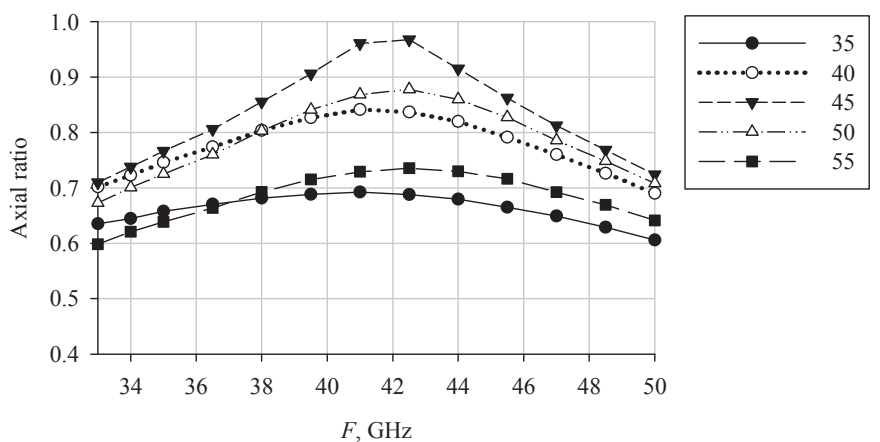

Fig. 3. Axial ratio dependence of the spherical flat-layered lens for different incline angles of the polarization plane
For a case when the excitation wave is linearly polarized and the incline angle of electric field vector is $45^{\circ}$, the electromagnetic wave polarization in the lens aperture is close to circular at the frequency about $42 \mathrm{GHz}$. From the Fig. 3 it can be seen that the polarization plane angle strongly affects the axial ratio maximum value and slightly changes the position of the maximum on frequency axis. The position of the axial ratio maximum on frequency axis is determined by the lens electrical size - i.e., it depends on the path length which required for wave components with horizontal and vertical polarization to reach phase difference about $90^{\circ}$ at the lens output. Fig. 4 shows the dependence of axial ratio for lenses of various sizes from 0.9 up to 1.1 of the first lens diameter studied in $10 \%$ increments. The polarization plane angle in every case is fixed at $45^{\circ}$.

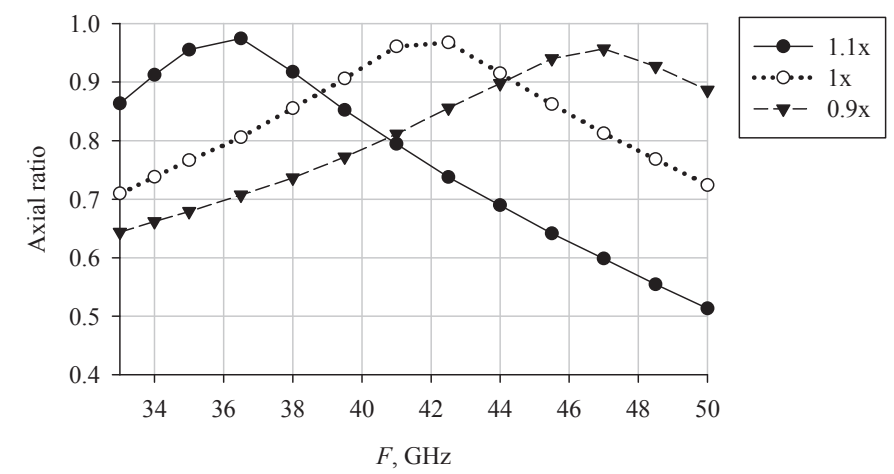

Fig. 4. Axial ratio dependence of the spherical flat-layered lens for different lens sizes

\section{Axial RATIO AND ScAn ANGLES}

The axial ratio dependence on the angle of the lens illumination was calculated for scan angles from $0^{\circ}$ to $30^{\circ}$ in the plane $Y O Z$, as it is shown in Fig. 5. Examination for scanning in the orthogonal plane does not make sense, because in this case electromagnetic wave propagation conditions are the same for any angle of illumination.

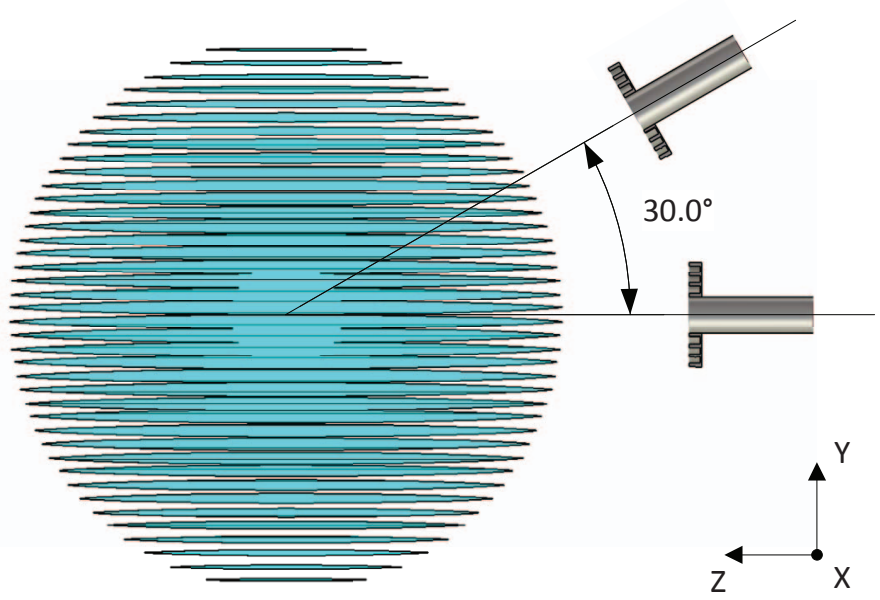

Fig. 5. Different angles of flat-layered lens illumination 
There is a strong dependence of the axial ratio on the illumination angle of flat-layered spherical lens. The dependences for investigated lenses are given in Fig. 6 for the scanning range designated in Fig. 5 with a step of $10^{\circ}$. The polarization plane angle for all dependencies is equal to $45^{\circ}$.

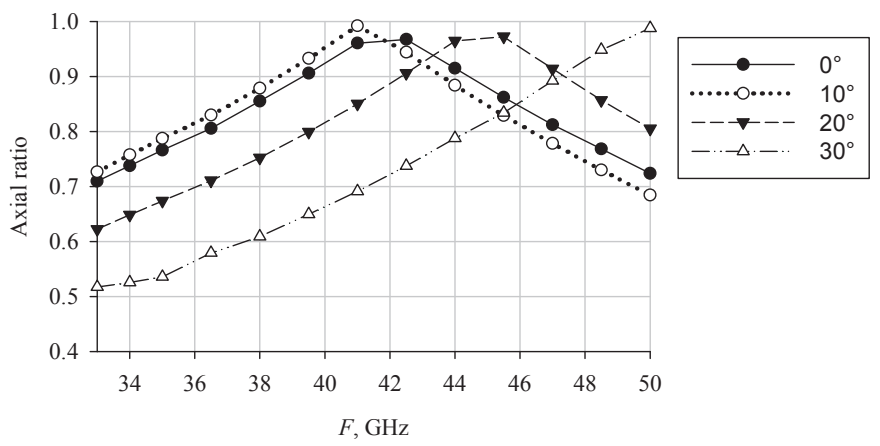

Fig. 6. Axial ratio dependences of the spherical flat-layered lens for different angles of illumination

The analysis of Fig. 6 shows that there is some non-linear behavior of the axial ratio maximum value depending on the lens illumination angle. It can be seen that in comparison with the reference case for the angle of $10^{\circ}$ the axial ratio maximum is located at lower frequency and for illumination angles of $20^{\circ}$ and $30^{\circ}$ maximums are at upper frequency. We can make a suggestion that nonlinearity is observed as a result of imprecise choice of the polarization plane angle. As the step of the angle variation is $5^{\circ}$ in this paper then the direction for the reference case may not coincide to the maximum values of axial ratio.

\section{CONCLUSION}

The results of the above studies show the possibilities and limitations for use of flat-layered spherical lens as an antenna with elliptical polarization which operates under illumination of linearly polarized wave. For example, in the frequency range $42.5-43.5 \mathrm{GHz}$ for scan angles of $\pm 20^{\circ}$ in the plane $Y 0 Z$ the possibility of axial ratio high values $(>0,9)$ within the main lobe is demonstrated. It is possible to choose frequency where the conversion of the feed horn linear (slant) polarization in circular polarization in the lens aperture is performed. This choice is realized by proper specifying of the lens size. On the other hand, the axial ratio strongly depends on the lens size and this fact is a limitation. Changing of the lens size will affect the overall gain of the antenna system, and it is possible only within certain limits.

\section{REFERENCES}

[1] Luneberg R.K. Mathematical Theory of Optics. Providence, Rhode Island: Brown University, 1944, p.189-213.

[2] Ryazantsev R.O., Alexandrin A.M., Salomatov Y.P., Sugak M.I. Artificial dielectric spherical Luneberg Lens. Russian Physics Journal., Tomsk State University publication, 2010, No 9/2, pp. 70-72.

[3] Ryazantsev R.O., Alexandrin A.M., Salomatov Y.P., Artificial dielectric quasioptical structure investigation at SHF range. Vestnik SibSAU. Aerospace tehnologies and control systems, vol. 6 (32), 2010, pp. 15-18.

[4] Ryazantsev R.O., Salomatov Y.P., Sugak M.I., Pavlon N.A., Sinitsin M.E. Multibeam broadband antenna system based on Luneberg lens. Nonlinear World, 2014, vol. 12, No 5, pp.37-44.

[5] Ryazantsev R.O., Salomatov Y.P. Patent No : RU 2485646. Focusing device of «Luneberg Lens» type. Date of Patent: March 2012.

[6] Ryazantsev R. O., Salomatov Y. P., Alexandrin A.M., Dranishnikov A. S. Circular polarization flat-layered spherical Luneberg Lens antenna system. Microwave \& Telecommunication Technology (CriMiCo), 2014 24th International Crimean Conference, 7-13 Sept. 2014, pp. 465 - 466. 